\title{
Caracterização do uso de medicamentos entre idosos cadastrados em uma unidade de Atenção Primária à Saúde de Diamantina, Minas Gerais, Brasil, 2011
}

\author{
Use of medication among elderly patients attended at Primary \\ Health Care Unit in Diamantina, Minas Gerais, Brazil, 2011
}

Hellen Lilliane CRUZ ${ }^{1}$, Flávia Karla Cruz MOTA², Renata Aline ANDRADE ${ }^{1}$,

Emerson Cotta BODEVAN ${ }^{3}$, Lorena Ulhôa ARAÚ JO ${ }^{1}$, Delba Fonseca SANTOS ${ }^{1}$ ${ }^{1}$ Departamento de Farmácia da Faculdade de Ciências Biológica e da Saúde; ${ }^{3}$ Departamento de Matemática e Estatística da Faculdade de Ciências Exatas e Tecnológica. Universidade Federal dos Vales Jequitinhonha e Mucuri. Rodovia MGT 367 - Km 583, $N^{\circ}$ 5000. Bairro Alto da Jacuba, Diamantina, Minas Gerais, Brasil. CEP-39100-000l. ${ }^{2}$ Prefeitura Municipal de Diamantina. Rua da Gloria- $n^{\circ}$ 394, Diamantina, Minas Gerais, Brasil CEP:39100-000.Email: hellen.crz@gmail.com

\begin{abstract}
This study aims to characterize the access and use of medications among elderly enrolled in a Primary Health Care Unit in Diamantina, Minas Gerais, Brazil. Descriptive study with elderly (aged 60 years or older) enrolled in a health unit in the period January to December 2011, with the realization of home interviews and application of semi-structured and standardized questionnaires. The average of medications consumed was 2.6 per elderly and polypharmacy was evidenced in $18.3 \%$ of cases. $82.5 \%$ of prescription medications were free provided in the public system of municipality, and $64.2 \%$ of these were for the cardiovascular system. Cardiovascular medications were the most commonly used class (diuretic and rennin-angiotensin system with $61.3 \%$ ). Thus the results of this study were comparable to those found in other research on medications use of drugs by elderly, confirming the importance the using of medicines in the elderly health care.
\end{abstract}

Key Words: Elderly; Polypharmacy; Primary Health Care; Descriptive Epidemiology

\section{RESUMO}

Este estudo visa caracterizar o acesso e uso de medicamentos entre os idosos cadastrados em uma Unidade de Atenção Primária à Saúde, em Diamantina, Estado de Minas Gerais, Brasil. Estudo descritivo com idosos (idade igual ou superior a 60 anos) cadastrados na unidade de saúde no período de janeiro a dezembro de 2011, com a realização de entrevistas domiciliares e aplicação de um questionário semi-estruturado e padronizado. A média de medicamentos consumidos foi 2,6 por idoso e a polifarmácia evidenciada em 18,3\% dos casos. Dos medicamentos prescritos, $82,5 \%$, foram dispensados gratuitamente no sistema público do município, destes $64,2 \%$ eram para o sistema cardiovascular. Os cardiovasculares foram à classe de medicamentos mais utilizada (diuréticos e sistema renina angiotensina com $61,3 \%$ ). Desta forma os resultados apresentados foram comparáveis ao encontrado em outras pesquisas sobre o uso de medicamentos por idosos, confirmando a importância dos medicamentos na atenção à saúde do idoso.

Palavras Chave: Idoso; Polifarmácia; Atenção Primária à Saúde; Epidemiologia Descritiva. 


\section{INTRODUÇÃO}

O Brasil vem experimentando, desde a criação do Sistema Único de Saúde (SUS), mudanças norteadas pela universalidade do acesso, integralidade da atenção e equidade por meio da Atenção Primária à Saúde (APS) (1), mostrando o impacto positivo da APS na saúde da população, a maior satisfação dos usuários e os menores custos para o sistema de saúde (2).

Nesta perspectiva, o aumento da população idosa no Brasil nas últimas décadas, exigiu do SUS o desenvolvimento e a execução de políticas, estratégias e ações que atendessem às necessidades específicas, contribuindo para que mais pessoas alcançassem a idade avançada com o melhor estado de saúde possível (3). Isso se deve ao fato de que a melhoria das condições de vida, a redução da fecundidade, o aumento da expectativa de vida e a utilização de novas técnicas de diagnósticos e métodos terapêuticos levaram a um aumento na demanda aos serviços de saúde constituída por pessoas com mais de 60 anos de idade (4).

No entanto, as pessoas idosas apresentam características específicas do ponto de vista fisiológico, psicológico e social, decorrentes das alterações no estado de saúde, e o consumo de medicamentos acompanha esta tendência (5), sendo possivelmente, o grupo etário mais medicalizado na sociedade, devido ao aumento da prevalência de doenças crônicas (6). O medicamento embora necessário em muitas ocasiões, quando mal utilizados podem desencadear complicações sérias para a saúde (7).

A Organização Mundial da Saúde (OMS) (8), destacou que mais de $50 \%$ de todos os medicamentos são incorretamente prescritos, dispensados e vendidos; mais de $50 \%$ dos pacientes os usam incorretamente; e mais de $50 \%$ de todos os países não implementam políticas básicas para promover o uso racional de medicamentos, de acordo com diretrizes clínicas.

No Brasil, um estudo realizado no município de Bambuí (6), mostrou o consumo elevado de medicamentos: entre 1.606 idosos, 1.383 (86\%) haviam utilizados no mínimo um medicamento no período de três meses. Ainda quanto ao consumo de medicamentos por idosos, Medeiros-Souza et al (2007) (9) verificaram que nos Estados Unidos da América o referido consumo aumentou por volta de $8,5 \%$ de 1991 a 2000.

Assim, o aumento do risco e da gravidade dos Problemas Relacionados aos Medicamentos (PRM), tais como as reações adversas, interações medicamentosas e erros de medicação, que podem reduzir a adesão ao tratamento e elevar a morbimortalidade está associada ao elevado consumo de medicamentos por idosos. Sendo a frequência dos PRM maior nesta faixa etária, aumentando expressivamente de acordo com a complexidade da terapia. O risco de ocorrência aumenta em $13 \%$ com o uso de dois agentes, em $58 \%$ quando este número aumenta para cinco, elevando-se para $82 \%$ nos casos em que são consumidos sete ou mais medicamentos (10).

Uma importante estratégia para reduzir estes problemas é a adoção das diretrizes da Política Nacional de Medicamentos em 2001 e da Política Nacional de Assistência Farmacêutica em 2004, que constituem um dos elementos fundamentais para a efetiva implementação de ações capazes de promover a melhoria das condições da assistência à saúde da população, garantindo a necessária segurança, eficácia e qualidade dos medicamentos, a promoção do uso racional e o acesso da população àqueles considerados essenciais $(11,12)$.

Diante da alta vulnerabilidade dos idosos à terapia medicamentosa e a complexidade dos problemas clínicos, compreender os padrões de utilização dos mesmos é essencial para avaliar o impacto das novas terapias, planejar e estabelecer melhorias nos serviços de APS (13), que devem realizar um acompanhamento contínuo a fim de proporcionar um tratamento eficaz na prevenção de possíveis complicações. Este estudo teve como objetivo descrever o perfil sociodemografico e caracterizar o uso de medicamento por idosos cadastrados em uma unidade de APS, no município de Diamantina, Minas Gerais.

\section{MÉTODO}

Trata-se de um estudo descritivo que utilizou como instrumento de coleta de informação um questionário semiestruturado e adaptado (14), para estudar os idosos cadastrados $(n=369)$ em uma unidade de APS do município de Diamantina, Minas Gerais, entre janeiro e dezembro de 2011, de acordo com os dados obtidos no Sistema de Informação de Atenção Básica (15).

Segundo o Instituto Brasileiro de Geografia e Estatística (16), no ano de 2010, Diamantina possuía uma área territorial $3.891,7 \mathrm{~km}^{2}$, densidade demográfica $11,79 \mathrm{hab} / \mathrm{km}^{2}$, pertence à mesorregião Jequitinhonha $\mathrm{e}$ o Índice Desenvolvimento Humano de 0,748 , população total 45.880 de habitantes, 40.064 residentes na área urbana $(87,3 \%)$ e 5.944 na área rural $(12,9 \%)$, taxa anual de crescimento $(0,45 \%)$. De acordo com o Cadastro Nacional de Estabelecimentos de Saúde (2010) (17), o município possuía oito unidades de APS na área urbana 
e quatro de Programa de Agentes Comunitários de Saúde (PACS) na rural, com cobertura de 59,38\% e 26,91\% respectivamente.

O questionário foi estruturado contendo questões abertas e fechadas, que abordaram aspectos socioeconômicos, uso de medicamentos, automedicação, condições de saúde e reações adversas, codificadas de forma padronizada em um banco de dados pelo programa Epi Info versão 6.04 (18).

O critério de seleção da unidade de APS estudada considerou que a mesma foi reestruturada pelo Programa Nacional de Reorientação da Formação Profissional em Saúde, em 2008, parceria da Universidade Federal dos Vales do Jequitinhonha e Mucuri (UFVJM), Ministério da Saúde (MS) e Secretaria Municipal de Saúde de Diamantina. A unidade é cenário de prática para o Programa de Educação pelo Trabalho em Saúde (PET-Saúde) com acadêmicos dos cursos de Enfermagem, Farmácia, Fisioterapia, Nutrição e Odontologia. A unidade de saúde possuia seis microáreas, uma equipe composta por um médico, uma enfermeira, dois técnicos de enfermagem e seis agentes comunitários para 3.388 pessoas. A inserção da Assistência Farmacêutica (AF) na unidade foi implementada com o Núcleo de Apoio à Saúde da Família (NASF) em 2011, com uma equipe multiprofissional, incluindo o farmacêutico (19).

Os critérios de inclusão no estudo foram: ter idade maior ou igual a 60 anos, estar cadastrados na unidade de APS e ter feito o uso de pelo menos um medicamento nos últimos 15 dias. Consideraram-se perdas: ausência por viagem; não localizados após quatro visitas; recusa por motivo pessoal. Idosos com problemas cognitivos tiveram o questionário respondido por cuidadores/responsáveis.

As variáveis de análise foram: socioeconômicas e demográficas (gênero, idade, estado civil, escolaridade, renda); uso de pelo menos um medicamento nos últimos 15 dias, sendo solicitada a apresentação da embalagem e/ou a prescrição médica. A prescrição identificou a utilização de medicamento de uso contínuo para tratar as morbidades referidas. O medicamento de uso contínuo é o consumido todos ou quase todos os dias, sem data para a interrupção da medicação. Na ausência da embalagem e/ou prescrição, era anotada o nome comercial do medicamento ou principio ativo segundo relato do entrevistado. O consumo foi estimado pelo número de entrevistados que relatou o uso de pelo menos um medicamento nos últimos 15 dias dividido pelo total de entrevistados. Dado que o número total de medicamentos consumidos não apresentou uma distribuição normal, essa variável foi categorizada em quartis, preservando a ausência de consumo como uma categoria em separado.
Foi utilizada a Relação de Medicamentos Essencial do Estado (RESME) vigente em 2011, para a identificação dos medicamentos padronizados ou não (20). A classificação e padronização dos medicamentos conforme substância ativa e indicação de uso foram conforme critérios estabelecidos pelo sistema de classificação Anatomical Therapeutic Chemical Index (ATC) (21), adotada pela OMS. O acesso aos medicamentos foi definido como a inclusão do mesmo à lista de medicamentos disponibilizados pelo município através da Farmácia Básica do SUS.

Para analise dos dados foi utilizado software (18), e para caracterizar o acesso e uso aos medicamentos, as seguintes variáveis foram utilizadas: número de medicamentos prescritos e não prescritos e forma de obtenção. Os dados foram apresentados na forma de tabelas, em frequência percentual simples, média e desvio padrão. Os testes qui-quadrado de Pearson e o exato de Fisher foram usados para testar a associação entre as características sócio demográficas dos idosos e o uso de medicamentos prescritos ou não prescritos.

O presente estudo faz parte de uma pesquisa maior, que inclui desfechos não mencionados neste artigo. Este estudo foi aprovado pelo Comitê de Ética em Pesquisa da UFVJM ( $n^{\circ}$ de registro 085/11). Os participantes do estudo foram orientados sobre o objetivo e procedimentos da pesquisa e assinaram o Termo de Consentimento Livre e Esclarecido.

\section{RESULTADOS E DISCUSSÃO}

Foram entrevistados 306 (82,9\%) idosos cadastrados na unidade de APS. A Tabela 1 resume as principais características sociodemográficas da população analisada. A média de idade dos idosos foi de 70,5 anos, com desvio padrão (DP) de 8,9. Em torno de $50 \%$ dos homens e das mulheres apresentaram escolaridade de até quatro anos. Mais da metade dos homens $(56,2 \%)$ e das mulheres $(55,4 \%)$ possuíam até dois salários mínimos.

Com relação ao uso do serviço de atenção em saúde, $284(92,8 \%)$ participaram de zero a quatro consultas/ ano; 22 (7,2\%) de cinco até 12 consultas/ano e $27(8,8 \%)$ idosos foram hospitalizados nos últimos 12 meses. Além disso, dos 306 idosos, auto referiram as seguintes condições de saúde: 159 (52,0\%) hipertensão arterial sistêmica, $86(28,1 \%)$ diabetes mellitus e $44(14,4 \%)$ queixas de dor (cefaleia).

A frequência do uso de medicamentos na população estudada foi igual a 78,1\%. O número médio de medicamentos consumidos foi igual a 2,6 $(\mathrm{DP}=2,1)$. 
Tabela 1 - Distribuição das variáveis sócio-demográficas entre os idosos com mais de 60 anos, segundo o gênero,Unidade de Atenção Primária (APS)/Diamantina, Minas Gerais, Brasil, 2011. (n=306)

\begin{tabular}{|c|c|c|c|c|c|c|c|}
\hline Variáveis & & Mulheres & & & Homens & & $\mathbf{P}$ \\
\hline & N & & $\%$ & $\mathrm{~N}$ & & $\%$ & \\
\hline \multicolumn{8}{|l|}{ Idade (anos) } \\
\hline $60-69$ & 117 & & 57,6 & 59 & & 57,3 & \\
\hline $70-79$ & 43 & & 21,2 & 31 & & 30,1 & $0,149^{*}$ \\
\hline $80-89$ & 33 & & 16,2 & 10 & & 9,7 & \\
\hline 90 ou + & 11 & & 5,4 & 3 & & 2,9 & \\
\hline \multicolumn{8}{|c|}{ Escolaridade (anos de estudo) } \\
\hline 0 & 60 & & 29,5 & 19 & & 18,4 & \\
\hline $1-4$ & 52 & & 25,6 & 39 & & 37,8 & $0,078+$ \\
\hline $5-8$ & 55 & & 27,1 & 26 & & 25,2 & \\
\hline 9 ou + & 36 & & 17,7 & 19 & & 18,4 & \\
\hline \multicolumn{8}{|l|}{ Renda familiar (SM) } \\
\hline$\leq 1$ & 31 & & 15,3 & 12 & & 11,7 & \\
\hline $1,1-2$ & 83 & & 40,9 & 36 & & 34,9 & $0,331+$ \\
\hline $2,1-3$ & 44 & & 21,7 & 23 & & 22,3 & \\
\hline 3,1 ou + & 45 & & 22,2 & 32 & & 31,1 & \\
\hline \multicolumn{8}{|l|}{ Estado Civil } \\
\hline Casado & 69 & & 34,0 & 75 & & 72,8 & $\leq 0,000+$ \\
\hline Solteiro/viúvo & 134 & & 66,0 & 28 & & 27,2 & \\
\hline Total & & 203 & & & 103 & & \\
\hline
\end{tabular}

SM:Salário Mínimovigenteàépoca da pesquisa $(1 S M=R \$ 510,00) ;+$ Testedequi-quadrado de Pearsone * Testeexato de Fisher, significativo quando $\leq \leq 0,05$.

Tabela 2 - Distribuição proporcional (\%) do uso de medicamentos e média do número de medicamentos utilizados entre idosos, segundo sexo e faixa etária. Unidade de Atenção

Primária (APS)/Diamantina, Minas Gerais, Brasil, 2011. $(\mathrm{n}=306)$

\begin{tabular}{|l|r|r|r|r|r|}
\multicolumn{1}{|c|}{ Gênero/Faixa etária } & Nenhum & $\mathbf{1 - 2}$ & $\mathbf{3 - 4}$ & $\mathbf{5 +}$ & Média \\
\hline Homens (anos) & & & & & 1,8 \\
\hline $60-69$ & 32,2 & 32,2 & 25,4 & 10,2 & 1,88 \\
\hline $70-79$ & 22,6 & 38,8 & 22,6 & 16,0 & 2,45 \\
\hline $80-89$ & 0,0 & 20,0 & 40,0 & 40,0 & 3,80 \\
\hline 90 ou + & 33,3 & 33,3 & 33,4 & 0,0 & 2,00 \\
\hline Todos & 26,2 & 33,0 & 26,2 & 14,6 & 2,24 \\
\hline Mulheres (anos) & & & & & \\
\hline $60-69$ & 23,3 & 31,1 & 27,6 & 22,7 & 2,50 \\
\hline $70-79$ & 18,6 & 21,0 & 39,5 & 20,9 & 2,86 \\
\hline $80-89$ & 6,3 & 31,3 & 37,5 & 24,9 & 3,50 \\
\hline 90 ou + & 18,2 & 36,4 & 27,3 & 18,1 & 3,00 \\
\hline Todos & 19,3 & 29,2 & 31,7 & 19,8 & 2,77 \\
\hline Homens e Mulheres (anos) & & & & & \\
\hline $60-69$ & 26,3 & 31,5 & 26,8 & 15,5 & 2,30 \\
\hline $70-79$ & 20,3 & 28,4 & 32,4 & 18,9 & 2,69 \\
\hline $80-89$ & 4,8 & 28,5 & 35,1 & 31,6 & 3,57 \\
\hline 90 ou + & 21,4 & 35,7 & 38,1 & 4,8 & 2,78 \\
\hline Todos & 21,6 & 30,4 & 29,7 & 18,3 & 2,59 \\
\hline
\end{tabular}


A frequência de uso de pelo menos um medicamento nos últimos 15 dias foi mais alta entre as mulheres em comparação aos homens ( $80,3 \%$ vs $73,8 \%)$, assim, como a média do número de medicamentos consumidos $(2,8$ vs 2,2 , respectivamente). O consumo elevado de medicamentos (5+) foi observado em 18,3\% dos idosos; esta proporção foi igual a $14,6 \%$ entre os homens e $19,8 \%$ entre as mulheres (Tabela 2).

O consumo de medicamentos relatado pelos idosos apresentou o seguinte padrão: $42,5 \%$ dos idosos relataram ter consumido exclusivamente medi- camentos prescritos por médicos; $43,8 \%$ referiram uso de medicamentos prescritos e não prescritos e $13,7 \%$ referiram ter utilizado exclusivamente medicamentos não prescritos nos últimos 15 dias. Os medicamentos não prescritos mais consumidos foram os analgésicos/antipiréticos $(54,4 \%)$, seguidos pelos que atuam sobre o sistema músculo esqueléticos $(20,9 \%)$ e o sistema respiratório $(17,6 \%)$. Na Tabela 3 está apresentada a distribuição do uso de medicamentos prescritos e não prescritos segundo algumas características sociodemográficas.

Tabela 3: Distribuição do uso de medicamentos prescritos e não prescritos entre os idosos, segundo variáveis sociodemográficas. Unidade de Atenção Primária (APS)/ Diamantina, Minas Gerais, Brasil, 2011. (n=282)

\begin{tabular}{|c|c|c|c|c|}
\hline Variável Sociodemográficas & $\begin{array}{l}\text { Uso apenas de } \\
\text { medicamentos não } \\
\text { prescritos }(n=44) \%\end{array}$ & $\begin{array}{l}\text { Uso simultâneo de } \\
\text { medicamentos prescritos e } \\
\text { não prescritos }(n=133) \%\end{array}$ & $\begin{array}{c}\text { Uso exclusivo de } \\
\text { medicamentos } \\
\text { prescritos }(n=105) \%\end{array}$ & $p^{*}$ \\
\hline \multicolumn{5}{|l|}{ Gênero } \\
\hline Masculino & 36,4 & 29,3 & 34,3 & \\
\hline Feminino & 63,6 & 70,7 & 65,7 & $0,588+$ \\
\hline \multicolumn{5}{|l|}{ Estado civil } \\
\hline Casado & 52,3 & 45,9 & 44,8 & \\
\hline Viúvo/Solteiro & 47,7 & 54,1 & 55,2 & $0,691+$ \\
\hline \multicolumn{5}{|l|}{ Faixa etária (anos) } \\
\hline $60-69$ & 72,7 & 55,6 & 51,4 & \\
\hline $70-79$ & 18,2 & 21,8 & 28,6 & $0,134^{*}$ \\
\hline $80-89$ & 4,5 & 18,8 & 14,3 & \\
\hline 90 ou + & 4,5 & 3,7 & 5,7 & \\
\hline \multicolumn{5}{|l|}{ Escolaridade (anos) } \\
\hline 0 & 11,4 & 28,6 & 29,5 & \\
\hline $1-4$ & 25,0 & 21,8 & 27,6 & $0,117+$ \\
\hline $5-8$ & 40,9 & 20,3 & 25,7 & \\
\hline 9 ou + & 22,7 & 18,0 & 17,1 & \\
\hline \multicolumn{5}{|l|}{ Renda familiar (SM) } \\
\hline$\leq 1$ & 15,9 & 14,3 & 11,4 & \\
\hline $1,1-2$ & 38,6 & 40,6 & 30,1 & \\
\hline $2,1-3$ & 18,2 & 21,8 & 20,9 & $0,939+$ \\
\hline $3 \mathrm{ou}+$ & 27,3 & 23,3 & 29,5 & \\
\hline \multicolumn{5}{|l|}{$\mathrm{N}^{\circ}$ de residentes no domicilio } \\
\hline 2 ou - & 27,3 & 27,8 & 19,0 & \\
\hline $3-4$ & 36,4 & 36,8 & 45,7 & $0,500+$ \\
\hline 5 ou + & 36,4 & 35,3 & 35,2 & \\
\hline
\end{tabular}

+Teste de qui-quadrado de Pearson, * teste exato de Fisher; significativo quando $p \leq 0,05$; SM: Salário mínimo vigente à época da pesquisa (1SM $=\mathrm{R} \$ 510,00)$. 
Tabela 4 - Descrição dos medicamentos prescritos no período da entrevista, conforme classificação anatômica (nível 1) e subgrupo terapêutico (nível 2) da Anatomical Terapeutic Chemical (ATC), segundo forma de obtenção. Unidade de Atenção Primária (APS)/ Diamantina, Minas Gerais, Brasil, 2011. (n=306)

\begin{tabular}{|c|c|c|c|c|c|c|}
\hline \multirow{2}{*}{ Medicamento } & \multicolumn{2}{|c|}{ SUS } & \multicolumn{2}{|c|}{ Compra } & \multicolumn{2}{|c|}{ Total } \\
\hline & $n$ & $\%$ & $\mathrm{n}$ & $\%$ & N & $\%$ \\
\hline C- Cardiovascular & 467 & 92,1 & 40 & 7,9 & 507 & 64,2 \\
\hline C03-Diurético & 160 & 93,0 & 12 & 7,0 & 172 & 21,8 \\
\hline Co9- Agentes sobre o sistema renina angiotensina & 132 & 95,0 & 7 & 5,0 & 139 & 17,6 \\
\hline C08- Bloqueadores do canal de cálcio & 46 & 85,2 & 8 & 14,8 & 54 & 6,8 \\
\hline C07- Betabloqueadores & 63 & 98,4 & 1 & 1,6 & 64 & 8,1 \\
\hline C01- Cardioterápicos & 12 & 66,7 & 6 & 33,3 & 18 & 2,3 \\
\hline C10- Antilipemicos & 29 & 96,7 & 1 & 3,3 & 30 & 3,8 \\
\hline CO2 - C04 - C05- Outros & 25 & 83,3 & 5 & 16,7 & 30 & 3,8 \\
\hline $\mathrm{N}$ - Sistema nervosa & 39 & 63,9 & 22 & 36,1 & 61 & 7,2 \\
\hline N06- Psicoanalépticos & 24 & 80,0 & 6 & 20,0 & 30 & 3,8 \\
\hline N05- Psicoleticos & 6 & 60,0 & 4 & 40,0 & 10 & 1,3 \\
\hline N03- Antiepileticos & 6 & 75,0 & 2 & 25,0 & 8 & 1,0 \\
\hline N02- Analgésicos & 3 & 25,0 & 9 & 75,0 & 12 & 1,5 \\
\hline N01- N04- N07- Outros & - & - & 1 & 100,0 & 1 & 0,1 \\
\hline A- Trato alimentar e metabolismo & 100 & 68,5 & 46 & 31,5 & 146 & 18,5 \\
\hline A10- Usados no diabetes & 79 & 65,8 & 41 & 34,2 & 120 & 15,2 \\
\hline A02-Antiácidos/anticulcerosos/antiflatulentos & 19 & 100,0 & - & - & 19 & 2,4 \\
\hline A11-Vitaminas & 1 & 100,0 & - & - & 1 & 0,1 \\
\hline ** Outros & 1 & 16,7 & 5 & 83,3 & 6 & 0,7 \\
\hline B- Sangue e órgãos formadores de sangue & 28 & 87,5 & 4 & 12,5 & 32 & 4,0 \\
\hline B01- Antiagregantes & 27 & 87,1 & 4 & 12,9 & 31 & 3,9 \\
\hline B03- Antianêmicos & 1 & 100,0 & - & - & 1 & 0,1 \\
\hline M- Sistema músculo esquelético & 3 & 15,0 & 17 & 85,0 & 20 & 2,5 \\
\hline M01- Anti-inflamatório e anti-reumáticos & - & - & 6 & 100,0 & 6 & 0,7 \\
\hline$* * *$ Outros & 3 & 21,4 & 11 & 78,6 & 14 & 1,8 \\
\hline H- Preparações hormonais sistêmicos, exceto hormônios sexuais & 9 & 90,0 & 1 & 10,0 & 10 & 1,2 \\
\hline H03-Terapêuticos tireoidianos & 7 & 87,5 & 1 & 12,5 & 8 & 1,0 \\
\hline H02- Outros & 2 & 100,0 & - & - & 2 & 0,2 \\
\hline J01- Antibacteriano de uso sistêmico & 4 & 80,0 & 1 & 20,0 & 5 & 0,6 \\
\hline D- Outros medicamentos & - & - & 1 & 100,0 & 1 & 0,1 \\
\hline Total & 652 & 82,5 & 138 & 17,5 & 790 & 100 \\
\hline
\end{tabular}

**A01-A03-A06; *** M03-M04.

As características gênero, estado civil, idade, escolaridade, renda familiar e número de residentes no domicílio, não apresentaram associações significantes $(\mathrm{p} \leq$ $0,05)$ com o consumo de medicamentos prescritos e não prescritos.

Entre os medicamentos prescritos por profissional do SUS, 91,6\% estão presentes na RENAME, e de acordo com os dados apresentados na Tabela 4, $82,5 \%$ na RESME, sendo $17,5 \%$ dos medicamentos obtidos no setor privado, ou seja, não constavam na RESME. Do total de 790 medicamentos consumidos, $64,2 \%$ atuavam sobre o sistema cardiovascular, $18,5 \%$ no trato alimentar e metabolismo e $7,2 \%$ no sistema nervoso. 
Inicialmente é importante destacar que esta pesquisa envolveu algumas limitações como o espaço geográfico limitado (abrangência de uma APS) e as dificuldades de se entrevistar o idoso. Para minimizar um possível viés de memória, foram adotados procedimentos padronizados para a coleta de dados que incluíram a solicitação da apresentação da prescrição, embalagem ou bula do medicamento referido, e ter sido realizada por um único entrevistador.

A realização de estudos abrangentes sobre a utilização de medicamentos por idosos, como o presente trabalho, constitui uma demanda importante em nosso país, uma vez que este tema ainda é insuficientemente abordado e os estudos realizados até o momento são restritos a alguns municípios brasileiros $(22,23,24)$, o que geralmente impossibilita a extrapolação dos resultados para outras regiões e para o país (22).

As características sociodemográficas dos indivíduos incluídos neste estudo refletem em geral o quadro da população idosa no Brasil $(23,25)$, havendo maior proporção de mulheres e de idosos com idade mais avançada nas regiões com melhor nível socioeconômico.

O consumo de medicamentos entre os idosos foi menor que o observado em uma área urbana de Fortaleza (23) $(80,3 \%)$ e maior que o encontrado $(72,1 \%)$ no estudo desenvolvido em Belo Horizonte, por Loyola Filho et al (2006) (26).

A maioria dos estudos com idosos mostrou que o número médio de medicamentos utilizados por pessoa é entre 3 a 3,69 (27,28). Porém em estudos realizados, em Fortaleza (23) e Belo Horizonte (26), foram observadas respectivamente, médias de 2,3 e 2,2, sugerindo que em regiões distintas do país existem diferenças no consumo de medicamentos. A média encontrada nesta pesquisa foi de 2,6 $(\mathrm{DP}=2,1)$ medicamentos por idosos, podendo ser considerada elevada. $\mathrm{O}$ acesso fácil às medicações e a falta de conscientização do uso de medidas não farmacológicas podem estar contribuindo para o consumo de medicamentos nesta população. Este elevado percentual de consumo confirma a importância dos medicamentos nos cuidados à saúde do idoso e consequentemente a parceria da equipe da APS e do NASF com o desenvolvimento de ações para a promoção do uso racional de medicamentos.

Mudanças na frequência do uso de medicamentos podem refletir diferenças entre as populações quanto ao estado de saúde, utilização de serviço, modelo de atenção à saúde, além de traços demográficos e culturais ligados ao consumo de medicamentos, como sexo, idade e propensão ao uso (29). O uso de medicamentos entre idosos residentes na área de abrangência da unidade de saúde estudada pode ser atribuído a um ou mais destes fatores. É preciso considerar ainda que o menor uso de medicamentos neste estudo pode estar relacionado ao maior período recordatório adotado (15 dias) em comparação ao de Fortaleza (23) (7 dias). Desta forma, tornam-se necessários estudos mais abrangentes para explicar as diferenças no consumo de medicamentos entre diferentes populações (30).

No estudo de Flores e Mengue (2005), 27\% dos idosos apresentaram polifarmácia (14), ou seja, um consumo de cinco ou mais medicamentos por pessoa, dado superior ao encontrado no estudo realizado por Loyola Filho et al (2006) (14,3\%) (26) e também no presente estudo (18,3\%). Com a progressão da idade, este fenômeno pode ser explicado por uma série de fatores, incluindo aumento das morbidades, o que poderá ocasionar ao uso de medicamentos inadequados, possibilitando a ocorrência de interações e reações adversas (31).

Quanto ao uso dos medicamentos prescritos entre os idosos estudados, quando comparado às listas de medicamentos selecionados pela RENAME e RESME, isto é, medicamentos padronizados como essenciais, foi constatado neste estudo que $82,5 \%$ estavam em consonância com estas e, portanto, com a Política Nacional de Medicamentos. O presente estudo mostrou que o acesso aos medicamentos prescritos estava abaixo dos resultados obtidos por Panis et al (2008) (32) (87\%) e por Aziz et al (2011) (33) (95,8\%).

Por outro lado, esse trabalho mostrou que os idosos utilizaram o sistema público de saúde para obter medicamentos, uma vez que $53,3 \%$ possuíam até dois salários mínimos e dois anos de escolaridade, perfil este, hegemonicamente, composto por população que depende do SUS para ter acesso aos medicamentos (33).

Neste aspecto, a adoção da lista de medicamentos essenciais constitui um importante instrumento de orientação das ações de saúde relacionado ao uso de medicamento, já que por meio destas orientações é possível garantir a necessária segurança, eficácia e qualidade dos medicamentos, a promoção do uso racional e o acesso da população àqueles considerados essenciais (9).

\section{CONCLUSÃO}

Estudos evidenciaram que os medicamentos de uso contínuo com ação sobre o aparelho cardiovascular são os mais consumidos por idosos $(28,30,34)$, resultados semelhantes foram observados no presente trabalho. O predomínio de medicamentos anti-hipertensivos - diuréticos e os que atuam sobre o sistema renina angiotensina - responderam por cerca de $61,3 \%$ do total, dado coerente com o fato da hipertensão ser a condição crônica mais frequente entre os idosos estudados.

Em resumo, os resultados deste trabalho sugerem que, de maneira geral, o acesso aos medicamentos é mui- 
to semelhante ao observado em outros estudos, apesar das distintas metodologias empregadas. Certamente não retrataram a totalidade dos medicamentos utilizados pelos idosos no município, uma vez que o estudo foi realizado em uma unidade de APS. No entanto, conseguiu demonstrar o elevado número de idosos que utilizaram medicamentos, as principais classes farmacológicas e a dependência do setor público para obtenção dos mesmos.

Em virtude disso, por meio dos múltiplos esforços de pesquisas na APS novos estudos de base populacional serão necessários, a fim de avaliar a consistência dos achados e, sobretudo para permitir uma compreensão de toda a complexidade do tema que envolve o papel do farmacêutico no NASF.

\section{AGRADECIMENTOS}

Os autores agradecem o apoio financeiro da Fundação de Amparo a Pesquisa do Estado de Minas Gerais - FAPEMIG - CDS-APQ-01907-08 e pela disponibilidade dos dados à Secretária Municipal de Saúde de Diamantina-MG.

\section{REFERÊNCIAS}

1. OPAS.Organização Pan-Americana da Saúde. O cuidado das condições crônicas na atenção primária à saúde: o imperativo da consolidação da estratégia da saúde da família/ Eugênio Vilaça Mendes. Brasília: Organização Pan-Americana da Saúde, 2012.

2. BRASIL. Conselho Nacional de Secretários de Saúde. Atenção Primária e Promoção da Saúde. Brasília: Conselho Nacional de Secretários de Saúde; 2007.

3. BRASIL. Ministério da Saúde. Secretaria de Atenção à Saúde. Departamento de Atenção Básica. Envelhecimento e saúde da pessoa idosa. Brasília: Ministério da Saúde, 2006.

4. Veras R, Lourenço R, Martins CSF, Sanchez MAS, Chaves PH. Novos paradigmas do modelo assistencial no setor saúde: consequência da explosão populacional dos idosos no Brasil. In: Veras RP. Terceira idade: gestão contemporânea em saúde. Rio de Janeiro: Relume Dumara/ UnATI/UERJ; 2002.

5. Lima-Costa MF. Epidemiologia do envelhecimento no Brasil. In: Rouquayrol MA, Almeida Filho N. Epidemio-

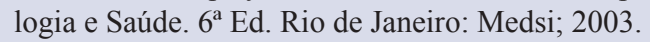

6. Loyola Filho AI, Uchoa E, Firmo JOA, Lima-Costa MF. Estudo de base populacional sobre o consumo de medicamentos entre idosos: Projeto Bambuí. Cad Saúde Pública. 2005; 21(2):545-53.

7. Barros JAC, Joany S. Anúncios de medicamentos em revistas médicas: ajudando a promover a boa prescrição? Ciên \& Saúde Coletiva 2002; 7:891-8.

8. WHO. World Health Organization. Medicines: rational use of medicines. Fact sheet $n^{\circ} 338$. May 2010. [cited 2010 set. 02]. Available from: http://www.who.int/mediacentre/factsheets/fs338/en/print.html.

9. Medeiros-Souza P, Santos-Neto LL, Kusano LTE, Pereira MG. Diagnosis and control of polypharmacy in the elderly. Rev Saúde Pública. 2007; 41(.6):1049-53.
10. Prybys KM, Melville K, Hanna J, Gee A, Chyka P. Polypharmacy in the elderly: clinical challenges in emergency practice: part 1 overview, etiology, and drug interactions. Emerg Med Rep. 2002; 23(8):145-53.

11. BRASIL. Ministério da Saúde. Secretaria de Políticas Públicas de Saúde. Política Nacional de Medicamentos. Brasília: Ministério da Saúde; 2001.

12. BRASIL. Ministério da Saúde. Resolução no 338, de 6 de maio de 2004. Aprova a Política Nacional de Assistência Farmacêutica. Diário Oficial da União, Brasília, DF, 20 de maio 2004, p. 52. [citado em 20 mai. 2013]. Disponível em: http://portal.saude.gov.br/portal/arquivos/pdf/ resol_cns338.pdf.

13. Chen YF, Dewey ME, Avery AJ; Analysis group of the MRCCFA study. Self-reported medication use for older people in England and Wales. J Clin Pharm Ther 2001; 26(2):129-40.

14. Flores LM, Mengue SS. Uso de medicamentos por idosos em região sul do Brasil. Rev Saúde Pública. 2005; 39(6):924-29.

15. BRASIL. Ministério da Saúde. Sistema de informação de Atenção Básica. [citado em 6 mar. 2013] Disponível em: http://www.datasus.gov.br/ SIAB/index.php?area=03.

16. IBGE. Instituto Brasileiro de Geografia e Estatísticas. Censo Demográfico 2010: resultados da amostra. [citado em 25 nov. 2012]. Disponível em: ftp.ibge.gov.br/Censos/ Censo_Demografico_2010/Resultados_do_Universo/tabelas_pdf/tab1.pdf

17. BRASIL. Ministério da Saúde. Sistema de Informação em Saúde. Cadastro Nacional de Estabelecimentos de Saúde. [citado em 10 mar. 2010] Disponível em: http//www. http://cnes.datasus.gov.br/.

18. Dean AG, Dean JA, Colombier D, Brendl KA, Smith DC, Burton $\mathrm{AH}$, et al. Epi Info: a word processing database and statistic program for epidemiolog on microcomputers. Atlanta: Center for Disease Control and Prevention; 1994. 
19. Prefeitura Municipal de Diamantina. Lei $n^{\circ} 3601$, de 05 de janeiro de 2011. Implanta, no âmbito do município de Diamantina, o Núcleo de Apoio à Saúde da Família (NASF), cria as funções que menciona e da outras providências. [citado em 18 mai. 2013]. Disponível em: http:// www.diamantina.mg.gov.br/sites/6600/6674/3601LeiIM PLANTA,NOAMBITODOMUNICIPIODEDIAMANTI NA,ONUCLEODEAPOIO.pdf.

20. SES-MG. Secretaria Estadual de Saúde de Minas Gerais. Rede Farmácia de Minas: Relação de Medicamentos do Estado de Minas Gerais. Versão Junho/2011.3ํe. Belo Horizonte: Secretária Estadual de Saúde de Minas Gerais; 2009.

21. WHO. World Health Organization. Collaborating Centre for Drug Statistics Methodology. Anatomical Therapeutic Chemical ATC/DDD Index 2011. Oslo: World Health Organization, 2011 [cited em 19 Mai. 2011]. Available from: http://www.whocc.no/atcddd/.

22. Silva $\mathrm{AL}$, Ribeiro $\mathrm{AQ}$, Klein $\mathrm{CH}$, Acurcio FA. Utilização de medicamentos por idosos brasileiros, de acordo com a faixa etária: um inquérito postal. Cad Saúde Pública, Rio de Janeiro, jun, 2012; 28(6):1033-45.

23. Coelho Filho JM, Marcopolito LF, Castelo A. Perfil de utilização de medicamentos por idosos em área urbana do Nordeste do Brasil. Rev Saúde Pública. 2004;38(4):557-64.

24. Dal Pizzol TS, Pons ES, Hugo FN, Bozzetti MC, Souza MLR, Hilgert JB. Uso de medicamentos entre idosos residentes em áreas urbanas e rurais de municípios no Sul do Brasil: um estudo de base populacional. Cad Saúde Pública, Rio de Janeiro. Jan, 2012, 28(1):104-14

25. Veras RP. País jovem com cabelos brancos: a saúde do idoso no Brasil. $2^{\text {a }}$ Ed. Rio de Janeiro:Relume Dumará; 1994.

26. Loyola Filho AI, Uchoa E, Lima Costa MF. Estudo epidemiológico de base populacional sobre uso de medicamentos entre idosos na Região Metropolitana de Belo Horizonte, Minas Gerais, Brasil. Cad Saúde Pública. 2006; 22(12):2657-67.
27. Teixeira JJV, Lefèvre F. A prescrição medicamentosa sob a ótica do paciente idoso. Rev .ijmkiimo,lçSaúde Pública. 2001; 35(2):207-13.

28. Bertoldi AD, Barros AJD, Hallsl PC, Lima RC. Utilização de medicamentos em adultos: prevalência e determinantes individuais. Rev de Saúde Pública. 2004; 38:228-38.

29. Helling DK, Lemke JH, Semla TP, Wallace RB, Lipson DP, Cornoni Huntley J. Medication use characteristics in the elderly: the Iowa 65+ Rural Health Study. J Am Geriatr Soe. 1987; 35:4-12.

30. Ribeiro AQ, Rozenfeld S, Klein CH, César CC, Acurcio FA. Inquérito sobre uso de medicamentos por idosos aposentados, Belo Horizonte, MG. Rev Saúde Pública. 2008; 42:724-32.

31. Acurcio FA, Silva AL, Ribeiro AQ, Rocha NP, Silveira $\mathrm{MR}$, Klein $\mathrm{CH}$, Rozenfeld S. Complexidade do regime terapêutico prescrito para idosos. Rev Assoc Med Bras. 2009; 55(4): 468-74.

32. Paniz VMV, Fassa AG, Facchini LA, Bertoldi AD, Piccini RX, Tomasi E, Thumé E, Silveira DS, Siqueira FV, Rodrigues MA. Acesso a medicamentos de uso contínio em adultos e idosos nas regiões Sul e Nordeste do Brasil. Cad Saúde Pública. 2008; 24(2):267-80.

33. Aziz MM, Calvo MC, Schneider IJC, Xavier AJ, d'Orsi E. Prevalência e fatores associados ao acesso a medicamentos pela população idosa em uma capital do sul do Brasil: um estudo de base populacional. Cad Saúde Pública. $2011 ; 27(10): 1939-50$.

34. Santos TRA, Lima DM, Nakatani AYK, Pereira LV, Leal GS, Amaral RG. Consumo de medicamentos por idosos, Goiânia, Brasil. Rev Saúde Pública. 2013; 17(1):94-103.

35. Paim J, Travasso C, Almeida C, Bahia L. The Brazilian health system: history, advances, and challenges. Lancet. 2011; 377:1778-97. 